\title{
FRACTIONAL TIME-FREQUENCY DISTRIBUTIONS
}

\author{
Soo-Chang Pei Min-Hung $\mathrm{Yeh}^{2}$ \\ ${ }^{1}$ Department of Electrical Engineering, National Taiwan University, Taipei, Taiwan, R. O. C. China \\ Email address: pei@cc.ee.ntu.edu.tw \\ ${ }^{2}$ Department of Electrical Engineering, National Taiwan University, Taipei, Taiwan, R. O. C. China \\ Email address: yeh@icsp.ee.ntu.edu.tw
}

\begin{abstract}
Several quadratic signal representation methods have been successfully used in signal processing. Four quadratic signal representations, Wigner distribution, Ambiguity function, signal correlation function and spectral correlation function are the traditional ones. In this paper, we propose a generalized distribution of the four quadratic time-frequency representations. The proposed distributions can have partial time/frequency-lag and partial time-lag/frequency characteristics of signals.
\end{abstract}

\section{INTRODUCTION}

Several quadratic signal representation methods are successfully used in signal processing[1]. Four basic quadratic signal representation methods are the prototypes of quadratic signal representation, and they are signal correlation function, spectrum correlation function, Wigner distribution and Ambiguity function. These four representations are defined as follows

- Signal Correlation Function

$$
C_{x}(t, \tau)=x\left(t+\frac{\tau}{2}\right) x^{*}\left(t-\frac{\tau}{2}\right)
$$

- Spectral Correlation Function

$$
S_{x}(\eta, \omega)=\mathcal{X}\left(\omega+\frac{\eta}{2}\right) \mathcal{X}^{*}\left(\omega-\frac{\eta}{2}\right)
$$

- Wigner Distribution

$$
\begin{aligned}
W_{x}(t, \omega) & =\int_{-\infty}^{\infty} x\left(t+\frac{\tau}{2}\right) x^{*}\left(t-\frac{\tau}{2}\right) e^{-j \omega \tau} d \tau \\
& =\int_{-\infty}^{\infty} \mathcal{X}\left(w+\frac{\eta}{2}\right) \mathcal{X}^{*}\left(w-\frac{\eta}{2}\right) e^{-j t \eta} d \eta(4)
\end{aligned}
$$

- Ambiguity Function

$$
\begin{aligned}
A_{x}(\eta, \tau) & =\int_{-\infty}^{\infty} x\left(t+\frac{\tau}{2}\right) x^{*}\left(t-\frac{\tau}{2}\right) e^{-j t \eta} d t \\
& =\int_{-\infty}^{\infty} \mathcal{X}\left(\omega+\frac{\eta}{2}\right) \mathcal{X}^{*}\left(\omega-\frac{\eta}{2}\right) e^{-j \omega \tau} d \omega(6)
\end{aligned}
$$

where $t$ is used to indicate the time variable, $\tau$ is the time-lag variable, $\omega$ is the frequency variable, and $\eta$ is the frequency-lag variable. The relationship of the above four signal representation methods are presented in [1]. Figure
1 quotes that the quadratic signal representations $C_{x}(t, \tau)$, $S_{x}(\eta, \omega), W_{x}(t, \omega)$ and $A_{x}(\eta, \tau)$ are interpreted by Fourier transforms.

Successive applications of the forward Fourier transform $\mathcal{F}$ on the signal $x(t)$ several times, and we will get $\mathcal{F}^{2}[x(t)]=x(-t), \mathcal{F}^{3}[x(t)]=\mathcal{X}(-w), \mathcal{F}^{4}[x(t)]=x(t)$. Based upon the above notation, the Fourier transform of signal can be interpreted as a $\frac{\pi}{2}$ angle rotation of signal in the time-frequency plane. A generalization of Fourier transform, FRFT, is developed and treated as a rotation of signal to any angles in the time-frequency plane. The transform kernel of continuous fractional Fourier transform (FRFT) is defined as follows [2][3] [4]:

$$
K_{\alpha}(t, u)=\sqrt{\frac{1-j \cot \alpha}{2 \pi}} e^{j \frac{t^{2}+u^{2}}{2} \cot \alpha-j u t \csc \alpha}
$$

where $\alpha$ indicates the rotation angle of transformed signal for FRFT. In [5], the 2D FRFT transform kernel with various orders in the two dimensions is defined as follows:

$$
\begin{aligned}
K_{\alpha, \beta}(s, t, u, v) \\
=\frac{1}{2 \pi} \sqrt{1-j \cot \alpha} \sqrt{1-j \cot \beta} e^{j \frac{s^{2}+u^{2}}{2} \cot \alpha-j s u \csc \alpha} . \\
\quad e^{j \frac{t^{2}+v^{2}}{2} \cot \beta-j t v \csc \beta}
\end{aligned}
$$

where $\alpha$ and $\beta$ indicate the rotation angles of the transformed signal for 2D FRFT.

\section{DEVELOPMENT OF FRACTIONAL TIME-FREQUENCY DISTRIBUTIONS}

If the Figure 1 is rotated by 45 degrees counterclockwise, a square can be obtained. We can find that there always exist a Fourier transform relation from $\tau$ to $\omega$ in the horizontal direction and another Fourier transform relation from $t$ to $\eta$ in the vertical direction. Moreover, we can generalize the Fourier transform with the fractional Fourier transform. Thus Figure 1 will become Figure 2. All the points located in the square of Figure 2 can have the time-frequency distributions. These distributions can have partial time and frequency-lag, partial time-lag and frequency characteristics of signal. They are called fractional time-frequency distributions. The fractional time-frequency distributions with parameter $\alpha_{1}, \alpha_{2}$ can be computed as:

$$
\Gamma_{x, \alpha_{1}, \alpha_{2}}(\nu, \mu)=\iint C_{x}(t, \tau) K_{\alpha_{1}, \alpha_{2}}(t, \tau, \nu, \mu) d t d \tau
$$

The traditional four quadratic signal representation are special cases of fraction time-frequency distribution, and they 
are located in the corners of the square. Four special cases of fractional time-frequency distributions can be obtained:

$$
\begin{aligned}
\Gamma_{x, 0,0}(\nu, \mu) & =C_{x}(\nu, \mu) \\
\Gamma_{x, 0, \frac{\pi}{2}}(\nu, \mu) & =W_{x}(\nu, \mu) \\
\Gamma_{x, \frac{\pi}{2}, 0}(\nu, \mu) & =A_{x}(\nu, \mu) \\
\Gamma_{x, \frac{\pi}{2}, \frac{\pi}{2}}(\nu, \mu) & =S_{x}(\nu, \mu)
\end{aligned}
$$

In [6], a definition of fractional Wigner distribution has been defined. The fractional Wigner distribution in [6] is a rotation of Wigner distribution in the time-frequency plane. The fractional time-frequency distribution defined here indicates partial time/frequency-lag and frequency/time-lag characteristics of signal. Figure 3 shows the different concepts of our fractional time-frequency distribution and Fractional Wigner distribution.

\section{PROPERTIES OF FRACTIONAL TIME-FREQUENCY DISTRIBUTIONS}

\section{- Time-shift}

For any signal $x(t)$, the signal correlation function of the time-lag signal $x(t-\lambda)$ can be computed as:

$$
C_{x}(t-\lambda, \tau)
$$

So the fractional time-frequency distributions of the time-lag signal can be computed as:

$$
\Gamma_{x, \alpha_{1}, \alpha_{2}}\left(\nu-\lambda \cos \alpha_{1}, \mu\right) e^{j \frac{\lambda^{2}}{2} \sin \alpha_{1} \cos \alpha_{1}-j \nu \lambda \sin \alpha_{1}}
$$

where $\Gamma_{x, \alpha_{1}, \alpha_{2}}(\nu, \mu)$ is the fractional time-frequency distributions of signal $x(t)$ with parameters $\left(\alpha_{1}, \alpha_{2}\right)$. The time-shift invariant property can be preserved while $\alpha_{1}=0$.

$$
\Gamma_{x, 0, \alpha_{2}}(\nu-\lambda, \mu)
$$

The frequency-shift property can be satisfied while $\alpha_{1}=\frac{\pi}{2}$.

$$
\Gamma_{x, \frac{\pi}{2}, \alpha_{2}}(\nu, \mu) e^{-j \nu \lambda}
$$

Figure 4 shows the angular scope of fractional timefrequency distribution for the time-shift signal that have time-shift and frequency-shift in distribution.

\section{- Frequency-shift}

For any signal $x(t)$, the signal correlation function of the frequency-shift signal $x(t) e^{j \lambda t}$ can be computed as:

$$
C_{x}(t, \tau) e^{j \lambda \tau}
$$

The fractional time-frequency distribution of the frequency-shift signal can be computed as:

$$
\Gamma_{x, \alpha 1, \alpha_{2}}\left(\nu, \mu-\lambda \sin \alpha_{2}\right) e^{-j \frac{\lambda^{2}}{2} \sin \alpha_{2} \cos \alpha_{2}+j \mu \lambda \cos \alpha_{2}}
$$

where $\Gamma_{x, \alpha_{1}, \alpha_{2}}(\nu, \mu)$ is the fractional time-frequency distributions of signal $x(t)$ with parameters $\left(\alpha_{1}, \alpha_{2}\right)$. The frequency-shift invariant property can be preserved while $\alpha_{2}=0$

$$
\Gamma_{x, \alpha_{1}, 0}(\nu, \mu) e^{j \mu \lambda}
$$

The time-shift property can be satisfied while $\alpha_{2}=\frac{\pi}{2}$.

$$
\Gamma_{x, \alpha_{1}, \frac{\pi}{2}}(\nu, \mu-\lambda)
$$

Figure 5 shows the angular scope of fractional timefrequency distribution for the frequency-shift signal that have time-shift and frequency-shift in distribution.
- Integration. in time/frequency-lag parameter If we calculate the integration of fractional timefrequency distribution in the time/frequency-lag variable, the following result is obtained:

$$
\int \Gamma_{x, \alpha_{1}, \alpha_{2}}(\nu, \mu) d \nu=\Gamma_{x, \alpha_{1}+\frac{\pi}{2}, \alpha_{2}}(0, \mu)
$$

Equation (18) indicates that the integration of time/frequency-lag variable for a fractional timefrequency distribution with parameter $\left(\alpha_{1}, \alpha_{2}\right)$ will cause the dc value of the distribution with parameter $\left(\alpha_{1}+\frac{\pi}{2}, \alpha_{2}\right)$. In the Wigner distribution case $\left(\alpha_{1}=0\right.$, $\alpha_{2}=\frac{\pi}{2}$ ), equation (18) becomes:

$$
\int \Gamma_{x, 0, \frac{\pi}{2}}(\nu, \mu) d \nu=\Gamma_{x, \frac{\pi}{2}, \frac{\pi}{2}}(0, \mu)=S_{x}(0, \mu)=\|X(\mu)\|^{2}
$$

We can verify that the Wigner distribution can preserve the frequency marginal propcrty[1]. In the Ambiguity function case $\left(\alpha_{1}=\frac{\pi}{2}, \alpha_{2}=0\right)$, equation (18) becomes:

$$
\int \Gamma_{x, \frac{\pi}{2}, 0}(\nu, \mu) d \nu=\Gamma_{x, \pi, 0}(0, \mu)=C_{x}(0, \mu)
$$

\section{- Integration in frequency/time-lag parameter}

If we calculate the integration of fractional timefrequency distribution in the frequency/time-lag variable, the following result is obtained:

$$
\int \Gamma_{x, \alpha_{1}, \alpha_{2}}(\nu, \mu) d \mu=\Gamma_{x, \alpha_{1}, \alpha_{2}+\frac{\pi}{2}}(\nu, 0)
$$

Equation (19) indicates that the integration of froquency/time-lag variable for a fractional timefrequency distribution with parameter $\left(\alpha_{1}, \alpha_{2}\right)$ will cause the $\mathrm{dc}$ value of the distribution with parameter $\left(\alpha_{1}, \alpha_{2}+\frac{\pi}{2}\right)$. In the Wigner distribution case $\left(\alpha_{1}=\right.$ $\left.0, \alpha_{2}=\frac{\pi}{2}\right)$, equation (19) becomes:

$$
\int \Gamma_{x, 0, \frac{\pi}{2}}(\nu, \mu) d \nu=\Gamma_{x, 0, \pi}(\nu, 0)=C_{x}(\nu, 0)=\|x(\nu)\|^{2}
$$

We can verify that the Wigner distribution can preserve the time marginal property[1]. In the Ambiguity function case $\left(\alpha_{1}=\frac{\pi}{2}, \alpha_{2}=0\right)$, equation (19) becomes:

$$
\int \Gamma_{x, \frac{\pi}{2}, 0}(\nu, \mu) d \nu=\Gamma_{x, \frac{\pi}{2}, \frac{\pi}{2}}(\nu, 0)=S_{x}(\nu, 0)
$$

\section{- Scaling}

For any signal $x(t)$, the fractional time-frequency distributions of the scaled signal $x(c t)$ can be computed as:

$$
\begin{aligned}
& \sqrt{\frac{1-j \cot \alpha_{1}}{c^{2}-j \cot \alpha_{1}}} \sqrt{\frac{1-j \cot \alpha_{2}}{c^{2}-j \cot \alpha_{2}}} e^{j \frac{\mu^{2}}{2} \cot \alpha_{1}\left(1-\frac{\cos ^{2} \beta_{1}}{\cos ^{2} \alpha_{1}}\right)} \\
& e^{j \frac{\mu^{2}}{2} \cot \alpha_{2}\left(1-\frac{\cos ^{2} \beta_{2}}{\cos ^{2} \alpha_{2}} \Gamma_{x, \beta_{1}, \beta_{2}}\left(\nu \frac{\sin \beta_{1}}{a \sin \alpha_{1}}, \mu \frac{\sin \beta_{2}}{a \sin \alpha_{2}}\right)\right.}
\end{aligned}
$$

where $\beta_{1}=\tan ^{-1}\left(a^{2} \tan \alpha_{1}\right)$ and $\beta_{2}=\tan ^{-1}\left(a^{2} \tan \alpha_{2}\right)$. $\Gamma_{x, \beta_{1}, \beta_{2}}$ is the fractional time-frequency distribution of 
signal $x(t)$ with angular parameters $\beta_{1}$ and $\beta_{2}$. While $\alpha_{1}=0$ and $\alpha_{2}=\frac{\pi}{2}$ (Wigner distribution), the distribution of the scaling signals becomes:

$$
W_{x}\left(c \nu,-\frac{\mu}{c}\right)
$$

While $\alpha_{2}=0$ and $\alpha_{1}=\frac{\pi}{2}$ (Ambiguity function), the distribution of the scaling signals becomes:

$$
A_{x}\left(-\frac{\nu}{c}, c \mu\right)
$$

\section{- Inverse Problem}

For any fractional time-frequency distribution with angular parameters $\left(\alpha_{1}, \alpha_{2}\right)$, the Signal correlation function $C_{x}(t, \tau)$ can be obtained by a 2D FRFT computation with angular parameter $\left(-\alpha_{1},-\alpha_{2}\right)$.

How to compute the signal from its Signal correlation function? There exist a strip $\tau=2 t$ in the $C_{x}(t, \tau)$. Taking the strip of $C_{x}(t, \tau)$ will reach the original signal.

$$
C_{x}(t, 2 t)=x(2 t) x^{*}(0)
$$

So the original signal can be recovered by the following equation:

$$
x(t)=\frac{C_{x}\left(\frac{t}{2}, t\right)}{x^{*}(0)}
$$

\section{APPLICATIONS}

In this section, we will introduce an application of the fractional time-frequency distribution: chirp signal detection. To begin with, we simplify the general chirp signal as: $e^{j \frac{\alpha}{2} t^{2}}$. The fractional time-frequency distribution of this chirp signal is equal to $e^{j\left(A \nu^{2}+B \nu \mu+C \mu^{2}\right)}$ :

$$
\begin{aligned}
A & =\frac{1}{2} \frac{-a^{2} \tan \alpha_{1}-\tan \alpha_{2}}{1-a^{2} \tan \alpha_{1} \tan \alpha_{2}} \\
B & =\frac{a \sec \alpha_{1} \sec \alpha_{2}}{1-a^{2} \tan \alpha_{1} \tan \alpha_{2}} \\
C & =\frac{1}{2} \frac{-\tan \alpha_{1}-a^{2} \tan \alpha_{2}}{1-a^{2} \tan \alpha_{1} \tan \alpha_{2}}
\end{aligned}
$$

The peaks are occurred while the phase of the distribution are equal to zero or multiples of $2 \pi$. Obviously, there exist infinite peaks in the distributions. We can find that the phase of the distribution is a quadratic function, and its shape is depended on the discriminant of quadratic function.

$$
\begin{cases}\Delta<0 & \text { ellipse } \\ \Delta=0 & \text { parabola } \\ \Delta>0 & \text { hyperbola }\end{cases}
$$

The discriminant of the phase of the distribution can be computed as:

$$
\Delta=B^{2}-4 A C=\frac{a^{2}-\tan \alpha_{1} \tan \alpha_{2}}{1-a^{2} \tan \alpha_{1} \tan \alpha_{2}}
$$

The condition to decide the shape of fractional timefrequency distribution can be simplified as:

- If $a>1$

$$
\begin{cases}\frac{1}{a^{2}}<\tan \alpha_{1} \tan \alpha_{2}<a^{2} & \text { Ellipse } \\ \frac{1}{a^{2}}>\tan \alpha_{1} \tan \alpha_{2} \text { or } \tan \alpha_{1} \tan \alpha_{2}>a^{2} & \text { Hyperbola }\end{cases}
$$

- If $a=1$

The discriminant will be equal to 1 except the case $\tan \alpha_{1} \tan \alpha_{2}=1$, so the shape of fractional timefrequency distribution of chirp function will be with hyperbola shapes.

- If $a<1$

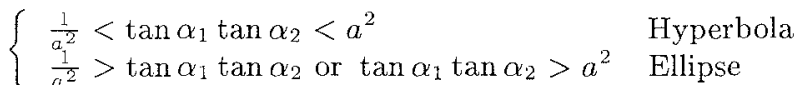

If the shapes of fractional time-frequency distribution are hyperbolas, the asymptotes are:

$$
\begin{aligned}
& \nu-\left(\csc \alpha_{2}+\cot \alpha_{2}\right) \mu=0 \\
& \nu-\left(\csc \alpha_{2}-\cot \alpha_{2}\right) \mu=0
\end{aligned}
$$

The geometric center is always located in the original point of $\mu \nu$ plane. The condition, $\tan \alpha_{1} \tan \alpha_{2}=1$, will cause a peaks in distribution, so it can be used to detect a chirp signal. Now, we analyze the generalized chirp signal: $e^{j\left(\frac{a}{2} t^{2}+b t\right)}$. Comparing the distribution of the generalized and simplified chirp signals, we can find that the fractional time-frequency distribution of a generalized chirp function is a translated version of the chirp signal $e^{j \frac{a}{2} t^{2}}$. The geometric center of distribution becomes $\left(-\frac{a b \sec \alpha_{2}}{a^{2}-\operatorname{tann} \alpha_{1} \tan \alpha_{2}},-\frac{b \sec \alpha_{1} \tan \alpha_{2}}{a^{2}-\tan \alpha_{1} \tan \alpha_{2}}\right)$. in the $\mu \nu$ plane. While the shapes of fractional distribution of a generalized chirp signal are hyperbolas, the asymptotes of the hyperbola are:

$$
\begin{aligned}
& \nu-m_{1} \mu-p=0 \\
& \nu-m_{2} \mu-q=0
\end{aligned}
$$

where

$$
\begin{aligned}
m_{1} & =\frac{-a \sec \alpha_{1} \sec \alpha_{2}+\sqrt{\left(a^{2}-\tan \alpha_{1} \tan \alpha_{2}\right)\left(1-a^{2} \tan \alpha_{1} \tan \alpha_{2}\right)}}{a^{2} \tan \alpha_{1}+\tan \alpha_{2}} \\
m_{2} & =\frac{-a \sec \alpha_{1} \sec \alpha_{2}-\sqrt{\left(a^{2}-\tan \alpha_{1} \tan \alpha_{2}\right)\left(1-a^{2} \tan \alpha_{1} \tan \alpha_{2}\right)}}{a^{2} \tan \alpha_{1}+\tan \alpha_{2}} \\
p & =\delta+\sigma \\
q & =\delta-\sigma \\
\delta & =\frac{b \sec \alpha_{1} \tan \alpha_{2}}{a^{2} \tan \alpha_{1}+\tan \alpha_{2}} \sqrt{\frac{a^{2} \tan \alpha_{1} \tan \alpha_{2}-1}{1-a^{2} \tan \alpha_{1} \tan \alpha_{2}}} \\
\sigma & =a b \frac{\tan \alpha_{1} \sec \alpha_{2}}{a^{2} \tan \alpha_{1}+\tan \alpha_{2}}
\end{aligned}
$$

Example:

In this example, we will compute the real part of the fractional time-frequency distribution of chirp signal $e^{j\left(t^{2}+t\right)}$. Figure 6 shows the fractional time-frequency distribution of the chirp signal. It can be found that the center of the distribution is located in $\left(-\sec \alpha_{2},-\tan \alpha_{2}\right)$. The peaks occurred while the angular parameters $\alpha_{1}=\frac{\pi}{6}$ and $\alpha_{2}=\frac{\pi}{3}$. So the parameter $a$ can be detected as 1 , and the parameter $b$ is also 1 .

\section{REFERENCES}

[1] F. Hlawatsch and G. F. Bourdeaux-Bartels, "Linear and quadratic time-frequency signal representations," IEEE Signal Processing Mag., vol. 9, pp. 21-67, Apr. 1992.

[2] A. C. McBride and F. H. Kerr, "On Namias' fractional Fourier transforms," IMA J. Appl. Math., vol. 39, pp. 159-175, 1987. 
[3] V. Namias, "The fractional order Fourier transform and its application to quantum mechanics," J. Inst. Maths Applics, vol. 25, pp. 241-265, 1980.

[4] L. B. Almeida, "The fractional Fourier transform and time-frequency representation," IEEE Trans. Signal Process., vol. 42, pp. 3084-3091, Nov. 1994.

[5] A. Sahlin, H. M. Ozaktas, and D. Mendlovic, "Optical implementation the two-dimensional fractional Fourier transform with different orders in the two dimensions." Optics Communications, vol. 120, pp. 134-138, 1995.

[6] D. Dragonman, "Fractional Wigner disbtribution function," J. Opt. Soc. Amer., A, vol. 13, pp. 474-478, Mar 1996.

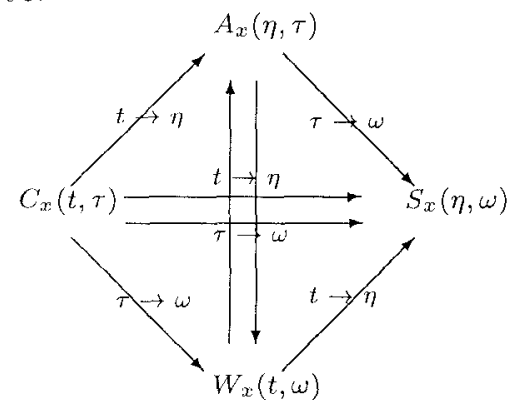

Figure 1. The quadratic signal representations represented by Fourier transforms

$$
A_{x}(\eta, \tau) \quad S_{x}(\eta, \omega)
$$

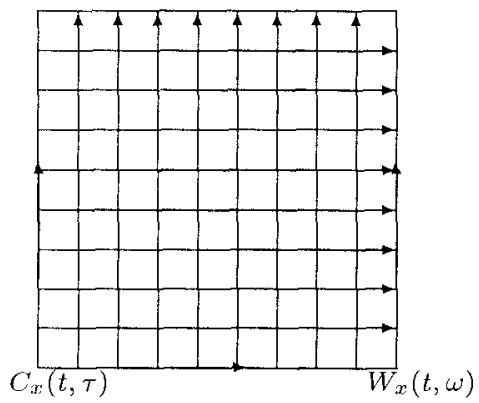

Figure 2. Quadratic signal representation and Fractional Time-Frequency distributions

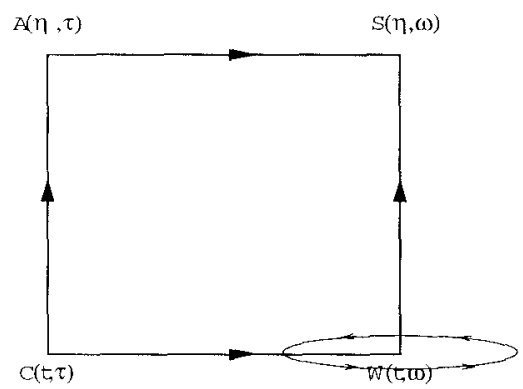

Figure 3. The different concepts of fractional Wigner distribution and fractional time-frequency distribution
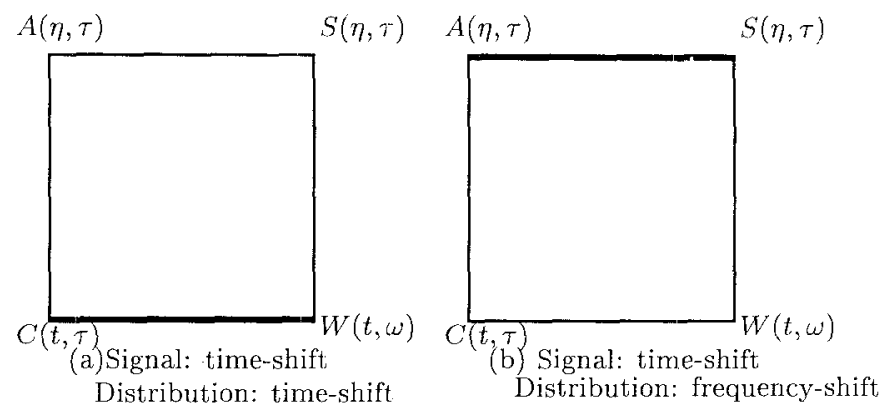

Figure 4. The angular scope of fractional timefrequency distribution for the time-shift property preserved
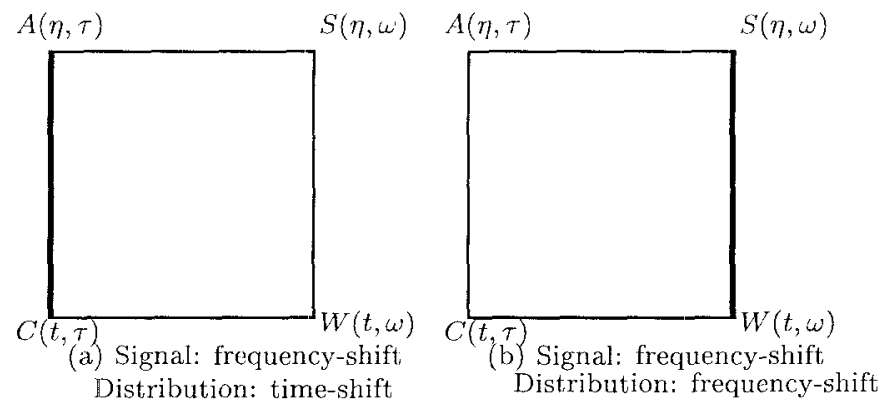

Figure 5. The angular scope of fractional timefrequency distribution for the time-shift property preserved
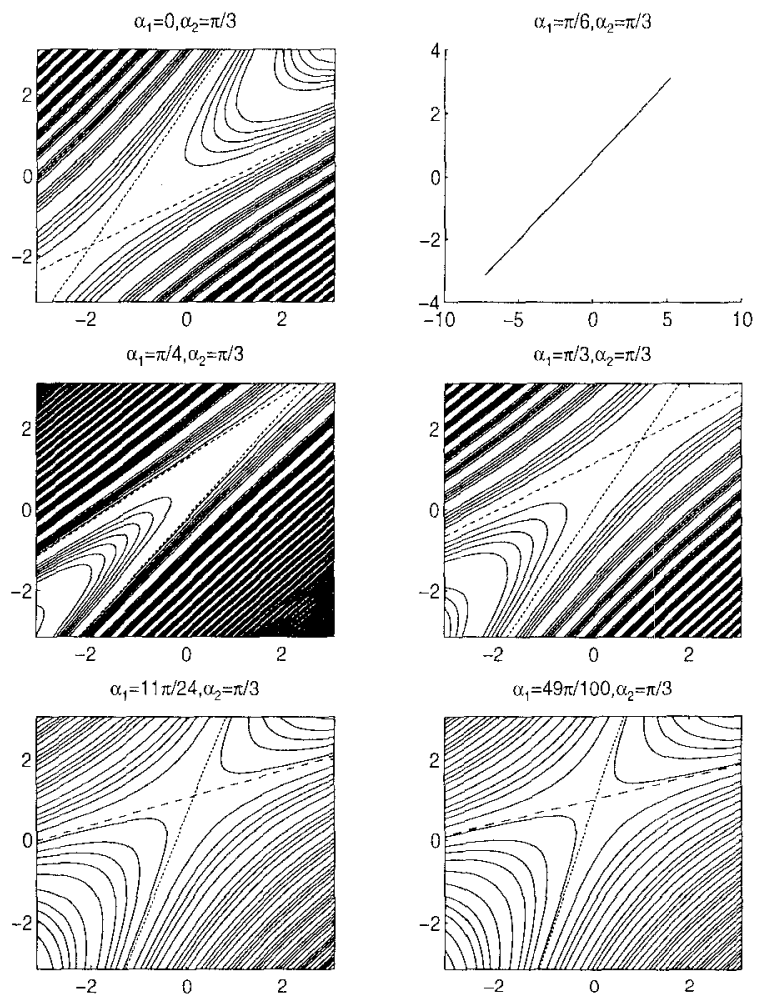

Figure 6. The fractional time-frequency distribution of the chirp signal 